\title{
Erratum: Search for new physics in events with same-sign dileptons and jets in pp collisions at $\sqrt{s}=8 \mathrm{TeV}$
}

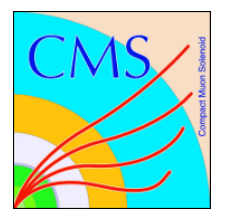

\section{The CMS collaboration}

E-mail: cms-publication-committee-chair@cern.ch

ERRATUM TO: JHEP01(2014)163

ARXIV EPRINT: 1311.6736

We update the parameters of the fitted functions for lepton and jet selection efficiencies presented in section 9 of the original paper. Due to an editorial error, these numbers were wrongly inserted. The corresponding figures, figures 10 and 11 of the original paper, showing the fitted functions, are unchanged.

\begin{tabular}{|l|cc|}
\hline Parameter & Electrons & Muons \\
\hline$\epsilon_{\infty}$ & $0.597 \pm 0.001$ & $0.617 \pm 0.001$ \\
$\epsilon_{10}$ & $0.133 \pm 0.002$ & $0.291 \pm 0.002$ \\
$\sigma(\mathrm{GeV})$ & $37.75 \pm 0.30$ & $29.95 \pm 0.38$ \\
\hline
\end{tabular}

Table 1. The parameters of the fit performed in figure 11 of the original paper for electron and muon selection efficiencies. This table replaces table 13 of the original paper.

The parameters of the fit shown in figure 10 (left) of the original paper are $\left(\epsilon_{\infty}, x_{1 / 2}\right.$, $\sigma)=(1.0,34.9 \mathrm{GeV}, 14.0 \mathrm{GeV})$.

Open Access. This article is distributed under the terms of the Creative Commons Attribution License (CC-BY 4.0), which permits any use, distribution and reproduction in any medium, provided the original author(s) and source are credited. 


\section{The CMS collaboration}

Yerevan Physics Institute, Yerevan, Armenia

S. Chatrchyan, V. Khachatryan, A.M. Sirunyan, A. Tumasyan

Institut für Hochenergiephysik der OeAW, Wien, Austria

W. Adam, T. Bergauer, M. Dragicevic, J. Erö, C. Fabjan ${ }^{1}$, M. Friedl, R. Frühwirth ${ }^{1}$, V.M. Ghete, C. Hartl, N. Hörmann, J. Hrubec, M. Jeitler ${ }^{1}$, W. Kiesenhofer, V. Knünz, M. Krammer ${ }^{1}$, I. Krätschmer, D. Liko, I. Mikulec, D. Rabady² , B. Rahbaran, H. Rohringer, R. Schöfbeck, J. Strauss, A. Taurok, W. Treberer-Treberspurg, W. Waltenberger, C.E. Wulz ${ }^{1}$

National Centre for Particle and High Energy Physics, Minsk, Belarus

V. Mossolov, N. Shumeiko, J. Suarez Gonzalez

\section{Universiteit Antwerpen, Antwerpen, Belgium}

S. Alderweireldt, M. Bansal, S. Bansal, T. Cornelis, E.A. De Wolf, X. Janssen, A. Knutsson, S. Luyckx, L. Mucibello, S. Ochesanu, B. Roland, R. Rougny, H. Van Haevermaet, P. Van Mechelen, N. Van Remortel, A. Van Spilbeeck

\section{Vrije Universiteit Brussel, Brussel, Belgium}

F. Blekman, S. Blyweert, J. D'Hondt, N. Heracleous, A. Kalogeropoulos, J. Keaveney, T.J. Kim, S. Lowette, M. Maes, A. Olbrechts, D. Strom, S. Tavernier, W. Van Doninck, P. Van Mulders, G.P. Van Onsem, I. Villella

Université Libre de Bruxelles, Bruxelles, Belgium

C. Caillol, B. Clerbaux, G. De Lentdecker, L. Favart, A.P.R. Gay, T. Hreus, A. Léonard, P.E. Marage, A. Mohammadi, L. Perniè, T. Reis, T. Seva, L. Thomas, C. Vander Velde, P. Vanlaer, J. Wang

\section{Ghent University, Ghent, Belgium}

V. Adler, K. Beernaert, L. Benucci, A. Cimmino, S. Costantini, S. Dildick, G. Garcia, B. Klein, J. Lellouch, J. Mccartin, A.A. Ocampo Rios, D. Ryckbosch, M. Sigamani, N. Strobbe, F. Thyssen, M. Tytgat, S. Walsh, E. Yazgan, N. Zaganidis

\section{Université Catholique de Louvain, Louvain-la-Neuve, Belgium}

S. Basegmez, C. Beluffi ${ }^{3}$, G. Bruno, R. Castello, A. Caudron, L. Ceard, G.G. Da Silveira, C. Delaere, T. du Pree, D. Favart, L. Forthomme, A. Giammanco ${ }^{4}$, J. Hollar, P. Jez, M. Komm, V. Lemaitre, J. Liao, O. Militaru, C. Nuttens, D. Pagano, A. Pin, K. Piotrzkowski, A. Popov ${ }^{5}$, L. Quertenmont, M. Selvaggi, M. Vidal Marono, J.M. Vizan Garcia

Université de Mons, Mons, Belgium

N. Beliy, T. Caebergs, E. Daubie, G.H. Hammad

Centro Brasileiro de Pesquisas Fisicas, Rio de Janeiro, Brazil

G.A. Alves, M. Correa Martins Junior, T. Martins, M.E. Pol, M.H.G. Souza 
Universidade do Estado do Rio de Janeiro, Rio de Janeiro, Brazil

W.L. Aldá Júnior, W. Carvalho, J. Chinellato ${ }^{6}$, A. Custódio, E.M. Da Costa, D. De Jesus Damiao, C. De Oliveira Martins, S. Fonseca De Souza, H. Malbouisson, M. Malek, D. Matos Figueiredo, L. Mundim, H. Nogima, W.L. Prado Da Silva, J. Santaolalla, A. Santoro, A. Sznajder, E.J. Tonelli Manganote ${ }^{6}$, A. Vilela Pereira

Universidade Estadual Paulista ${ }^{a}$, Universidade Federal do ABC ${ }^{b}$, São Paulo, Brazil

C.A. Bernardes ${ }^{b}$, F.A. Dias ${ }^{a}, 7$, T.R. Fernandez Perez Tomei ${ }^{a}$, E.M. Gregores $^{b}$, C. Lagana $^{a}$, P.G. Mercadante ${ }^{b}$, S.F. Novaes ${ }^{a}$, Sandra S. Padula ${ }^{a}$

Institute for Nuclear Research and Nuclear Energy, Sofia, Bulgaria

V. Genchev², P. Iaydjiev², A. Marinov, S. Piperov, M. Rodozov, G. Sultanov, M. Vutova University of Sofia, Sofia, Bulgaria

A. Dimitrov, I. Glushkov, R. Hadjiiska, V. Kozhuharov, L. Litov, B. Pavlov, P. Petkov

Institute of High Energy Physics, Beijing, China

J.G. Bian, G.M. Chen, H.S. Chen, M. Chen, R. Du, C.H. Jiang, D. Liang, S. Liang, X. Meng, R. Plestina ${ }^{8}$, J. Tao, X. Wang, Z. Wang

State Key Laboratory of Nuclear Physics and Technology, Peking University, Beijing, China

C. Asawatangtrakuldee, Y. Ban, Y. Guo, Q. Li, W. Li, S. Liu, Y. Mao, S.J. Qian, D. Wang,

L. Zhang, W. Zou

Universidad de Los Andes, Bogota, Colombia

C. Avila, C.A. Carrillo Montoya, L.F. Chaparro Sierra, C. Florez, J.P. Gomez, B. Gomez Moreno, J.C. Sanabria

Technical University of Split, Split, Croatia

N. Godinovic, D. Lelas, D. Polic, I. Puljak

University of Split, Split, Croatia

Z. Antunovic, M. Kovac

Institute Rudjer Boskovic, Zagreb, Croatia

V. Brigljevic, K. Kadija, J. Luetic, D. Mekterovic, S. Morovic, L. Tikvica

University of Cyprus, Nicosia, Cyprus

A. Attikis, G. Mavromanolakis, J. Mousa, C. Nicolaou, F. Ptochos, P.A. Razis

Charles University, Prague, Czech Republic

M. Finger, M. Finger Jr.

Academy of Scientific Research and Technology of the Arab Republic of Egypt, Egyptian Network of High Energy Physics, Cairo, Egypt

A.A. Abdelalim ${ }^{9}$, Y. Assran ${ }^{10}$, S. Elgammal ${ }^{9}$, A. Ellithi Kamel ${ }^{11}$, M.A. Mahmoud ${ }^{12}$, A. $\operatorname{Radi}^{13,14}$ 
National Institute of Chemical Physics and Biophysics, Tallinn, Estonia

M. Kadastik, M. Müntel, M. Murumaa, M. Raidal, L. Rebane, A. Tiko

Department of Physics, University of Helsinki, Helsinki, Finland

P. Eerola, G. Fedi, M. Voutilainen

Helsinki Institute of Physics, Helsinki, Finland

J. Härkönen, V. Karimäki, R. Kinnunen, M.J. Kortelainen, T. Lampén, K. Lassila-Perini,

S. Lehti, T. Lindén, P. Luukka, T. Mäenpää, T. Peltola, E. Tuominen, J. Tuominiemi,

E. Tuovinen, L. Wendland

Lappeenranta University of Technology, Lappeenranta, Finland

T. Tuuva

DSM/IRFU, CEA/Saclay, Gif-sur-Yvette, France

M. Besancon, F. Couderc, M. Dejardin, D. Denegri, B. Fabbro, J.L. Faure, F. Ferri,

S. Ganjour, A. Givernaud, P. Gras, G. Hamel de Monchenault, P. Jarry, E. Locci, J. Malcles, A. Nayak, J. Rander, A. Rosowsky, M. Titov

Laboratoire Leprince-Ringuet, Ecole Polytechnique, IN2P3-CNRS, Palaiseau, France

S. Baffioni, F. Beaudette, P. Busson, C. Charlot, N. Daci, T. Dahms, M. Dalchenko, L. Dobrzynski, A. Florent, R. Granier de Cassagnac, M. Haguenauer, P. Miné, C. Mironov, I.N. Naranjo, M. Nguyen, C. Ochando, P. Paganini, D. Sabes, R. Salerno, Y. Sirois, C. Veelken, Y. Yilmaz, A. Zabi

Institut Pluridisciplinaire Hubert Curien, Université de Strasbourg, Université de Haute Alsace Mulhouse, CNRS/IN2P3, Strasbourg, France

J.-L. Agram ${ }^{15}$, J. Andrea, D. Bloch, J.-M. Brom, E.C. Chabert, C. Collard, E. Conte ${ }^{15}$, F. Drouhin ${ }^{15}$, J.-C. Fontaine ${ }^{15}$, D. Gelé, U. Goerlach, C. Goetzmann, P. Juillot, A.-C. Le Bihan, P. Van Hove

Centre de Calcul de l'Institut National de Physique Nucleaire et de Physique des Particules, CNRS/IN2P3, Villeurbanne, France

S. Gadrat

Université de Lyon, Université Claude Bernard Lyon 1, CNRS-IN2P3, Institut de Physique Nucléaire de Lyon, Villeurbanne, France

S. Beauceron, N. Beaupere, G. Boudoul, S. Brochet, J. Chasserat, R. Chierici, D. Contardo, P. Depasse, H. El Mamouni, J. Fan, J. Fay, S. Gascon, M. Gouzevitch, B. Ille, T. Kurca, M. Lethuillier, L. Mirabito, S. Perries, J.D. Ruiz Alvarez ${ }^{16}$, L. Sgandurra, V. Sordini, M. Vander Donckt, P. Verdier, S. Viret, H. Xiao

E. Andronikashvili Institute of Physics, Academy of Science, Tbilisi, Georgia L. Rurua 


\section{RWTH Aachen University, I. Physikalisches Institut, Aachen, Germany}

C. Autermann, S. Beranek, M. Bontenackels, B. Calpas, M. Edelhoff, L. Feld, O. Hindrichs, K. Klein, A. Ostapchuk, A. Perieanu, F. Raupach, J. Sammet, S. Schael, D. Sprenger, H. Weber, B. Wittmer, V. Zhukov ${ }^{5}$

\section{RWTH Aachen University, III. Physikalisches Institut A, Aachen, Germany}

M. Ata, J. Caudron, E. Dietz-Laursonn, D. Duchardt, M. Erdmann, R. Fischer, A. Güth, T. Hebbeker, C. Heidemann, K. Hoepfner, D. Klingebiel, S. Knutzen, P. Kreuzer, M. Merschmeyer, A. Meyer, M. Olschewski, K. Padeken, P. Papacz, H. Pieta, H. Reithler, S.A. Schmitz, L. Sonnenschein, D. Teyssier, S. Thüer, M. Weber

\section{RWTH Aachen University, III. Physikalisches Institut B, Aachen, Germany}

V. Cherepanov, Y. Erdogan, G. Flügge, H. Geenen, M. Geisler, W. Haj Ahmad, F. Hoehle, B. Kargoll, T. Kress, Y. Kuessel, J. Lingemann², A. Nowack, I.M. Nugent, L. Perchalla, O. Pooth, A. Stahl

\section{Deutsches Elektronen-Synchrotron, Hamburg, Germany}

I. Asin, N. Bartosik, J. Behr, W. Behrenhoff, U. Behrens, A.J. Bell, M. Bergholz ${ }^{17}$, A. Bethani, K. Borras, A. Burgmeier, A. Cakir, L. Calligaris, A. Campbell, S. Choudhury, F. Costanza, C. Diez Pardos, S. Dooling, T. Dorland, G. Eckerlin, D. Eckstein, T. Eichhorn, G. Flucke, A. Geiser, A. Grebenyuk, P. Gunnellini, S. Habib, J. Hauk, G. Hellwig, M. Hempel, D. Horton, H. Jung, M. Kasemann, P. Katsas, C. Kleinwort, H. Kluge, M. Krämer, D. Krücker, W. Lange, J. Leonard, K. Lipka, W. Lohmann ${ }^{17}$, B. Lutz, R. Mankel, I. Marfin, I.-A. Melzer-Pellmann, A.B. Meyer, J. Mnich, A. Mussgiller, S. Naumann-Emme, O. Novgorodova, F. Nowak, J. Olzem, H. Perrey, A. Petrukhin, D. Pitzl, R. Placakyte, A. Raspereza, P.M. Ribeiro Cipriano, C. Riedl, E. Ron, M.Ö. Sahin, J. Salfeld-Nebgen, R. Schmidt ${ }^{17}$, T. Schoerner-Sadenius, M. Schröder, N. Sen, M. Stein, A.D.R. Vargas Trevino, R. Walsh, C. Wissing

\section{University of Hamburg, Hamburg, Germany}

M. Aldaya Martin, V. Blobel, H. Enderle, J. Erfle, E. Garutti, M. Görner, M. Gosselink, J. Haller, K. Heine, R.S. Höing, H. Kirschenmann, R. Klanner, R. Kogler, J. Lange, I. Marchesini, J. Ott, T. Peiffer, N. Pietsch, D. Rathjens, C. Sander, H. Schettler, P. Schleper, E. Schlieckau, A. Schmidt, T. Schum, M. Seidel, J. Sibille ${ }^{18}$, V. Sola, H. Stadie, G. Steinbrück, D. Troendle, E. Usai, L. Vanelderen

\section{Institut für Experimentelle Kernphysik, Karlsruhe, Germany}

C. Barth, C. Baus, J. Berger, C. Böser, E. Butz, T. Chwalek, W. De Boer, A. Descroix, A. Dierlamm, M. Feindt, M. Guthoff ${ }^{2}$, F. Hartmann², T. Hauth ${ }^{2}$, H. Held, K.H. Hoffmann, U. Husemann, I. Katkov ${ }^{5}$, A. Kornmayer ${ }^{2}$, E. Kuznetsova, P. Lobelle Pardo, D. Martschei, M.U. Mozer, Th. Müller, M. Niegel, A. Nürnberg, O. Oberst, G. Quast, K. Rabbertz, F. Ratnikov, S. Röcker, F.-P. Schilling, G. Schott, H.J. Simonis, F.M. Stober, R. Ulrich, J. Wagner-Kuhr, S. Wayand, T. Weiler, R. Wolf, M. Zeise 
Institute of Nuclear and Particle Physics (INPP), NCSR Demokritos, Aghia Paraskevi, Greece

G. Anagnostou, G. Daskalakis, T. Geralis, S. Kesisoglou, A. Kyriakis, D. Loukas, A. Markou, C. Markou, E. Ntomari, I. Topsis-giotis

University of Athens, Athens, Greece

L. Gouskos, A. Panagiotou, N. Saoulidou, E. Stiliaris

University of Ioánnina, Ioánnina, Greece

X. Aslanoglou, I. Evangelou, G. Flouris, C. Foudas, P. Kokkas, N. Manthos, I. Papadopoulos, E. Paradas

Wigner Research Centre for Physics, Budapest, Hungary

G. Bencze, C. Hajdu, P. Hidas, D. Horvath ${ }^{19}$, F. Sikler, V. Veszpremi, G. Vesztergombi ${ }^{20}$, A.J. Zsigmond

Institute of Nuclear Research ATOMKI, Debrecen, Hungary

N. Beni, S. Czellar, J. Molnar, J. Palinkas, Z. Szillasi

University of Debrecen, Debrecen, Hungary

J. Karancsi, P. Raics, Z.L. Trocsanyi, B. Ujvari

National Institute of Science Education and Research, Bhubaneswar, India

S.K. Swain ${ }^{21}$

Panjab University, Chandigarh, India

S.B. Beri, V. Bhatnagar, N. Dhingra, R. Gupta, M. Kaur, M.Z. Mehta, M. Mittal, N. Nishu, A. Sharma, J.B. Singh

University of Delhi, Delhi, India

Ashok Kumar, Arun Kumar, S. Ahuja, A. Bhardwaj, B.C. Choudhary, A. Kumar, S. Malhotra, M. Naimuddin, K. Ranjan, P. Saxena, V. Sharma, R.K. Shivpuri

Saha Institute of Nuclear Physics, Kolkata, India

S. Banerjee, S. Bhattacharya, K. Chatterjee, S. Dutta, B. Gomber, Sa. Jain, Sh. Jain, R. Khurana, A. Modak, S. Mukherjee, D. Roy, S. Sarkar, M. Sharan, A.P. Singh

Bhabha Atomic Research Centre, Mumbai, India

A. Abdulsalam, D. Dutta, S. Kailas, V. Kumar, A.K. Mohanty², L.M. Pant, P. Shukla, A. Topkar

Tata Institute of Fundamental Research - EHEP, Mumbai, India

T. Aziz, R.M. Chatterjee, S. Ganguly, S. Ghosh, M. Guchait ${ }^{22}$, A. Gurtu ${ }^{23}$, G. Kole, S. Kumar, M. Maity ${ }^{24}$, G. Majumder, K. Mazumdar, G.B. Mohanty, B. Parida, K. Sudhakar, N. Wickramage ${ }^{25}$

Tata Institute of Fundamental Research - HECR, Mumbai, India

S. Banerjee, S. Dugad 
Institute for Research in Fundamental Sciences (IPM), Tehran, Iran

H. Arfaei, H. Bakhshiansohi, H. Behnamian, S.M. Etesami ${ }^{26}$, A. Fahim ${ }^{27}$, A. Jafari, M. Khakzad, M. Mohammadi Najafabadi, M. Naseri, S. Paktinat Mehdiabadi, B. Safarzadeh ${ }^{28}$, M. Zeinali

\section{University College Dublin, Dublin, Ireland}

M. Grunewald

INFN Sezione di Bari ${ }^{a}$, Università di Bari ${ }^{b}$, Politecnico di Bari ${ }^{c}$, Bari, Italy

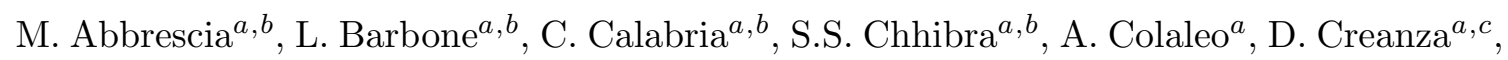
N. De Filippis ${ }^{a, c}$, M. De Palma ${ }^{a, b}$, L. Fiore ${ }^{a}$, G. Iaselli ${ }^{a, c}$, G. Maggi ${ }^{a, c}$, M. Maggi $^{a}$, B. Marangelli ${ }^{a, b}$, S. $\mathrm{My}^{a, c}$, S. Nuzzo ${ }^{a, b}$, N. Pacifico ${ }^{a}$, A. Pompili ${ }^{a, b}$, G. Pugliese ${ }^{a, c}$,

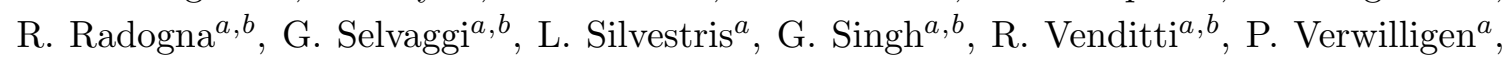
G. Zito ${ }^{a}$

\section{INFN Sezione di Bologna ${ }^{a}$, Università di Bologna ${ }^{b}$, Bologna, Italy}

G. Abbiendi $^{a}$, A.C. Benvenuti ${ }^{a}$, D. Bonacorsi ${ }^{a}, b$, S. Braibant-Giacomelli ${ }^{a, b}$, L. Brigliadori ${ }^{a, b}$, R. Campanini ${ }^{a, b}$, P. Capiluppi ${ }^{a, b}$, A. Castro ${ }^{a, b}$, F.R. Cavallo ${ }^{a}$, G. Codispoti ${ }^{a, b}$, M. Cuffiani ${ }^{a, b}$, G.M. Dallavalle ${ }^{a}$,F. Fabbri ${ }^{a}$, A. Fanfani ${ }^{a, b}$, D. Fasanella ${ }^{a}, b$, P. Giacomelli ${ }^{a}$, C. Grandi ${ }^{a}$, L. Guiducci ${ }^{a}, b$, S. Marcellini ${ }^{a}$, G. Masetti ${ }^{a}$, M. Meneghelli ${ }^{a}, b$,

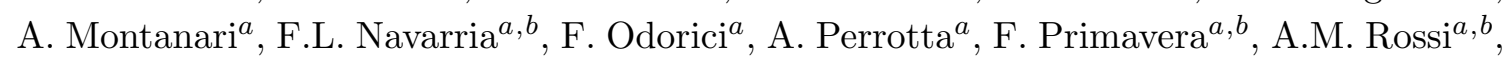
T. Rovelli ${ }^{a, b}$, G.P. Siroli ${ }^{a, b}$, N. Tosi ${ }^{a, b}$, R. Travaglini ${ }^{a, b}$

INFN Sezione di Catania ${ }^{a}$, Università di Catania ${ }^{b}, \operatorname{CSFNSM}^{c}$, Catania, Italy

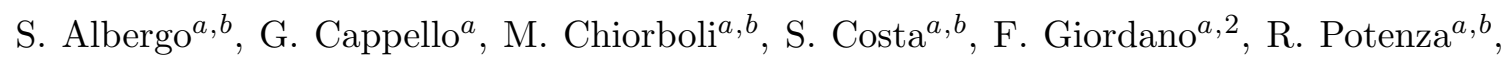
A. Tricomi ${ }^{a, b}$, C. Tuve ${ }^{a, b}$

INFN Sezione di Firenze ${ }^{a}$, Università di Firenze ${ }^{b}$, Firenze, Italy

G. Barbagli ${ }^{a}$, V. Ciulli ${ }^{a, b}$, C. Civinini ${ }^{a}$, R. D’Alessandro ${ }^{a, b}$, E. Focardi ${ }^{a, b}$, E. Gallo ${ }^{a}$, S. Gonzi ${ }^{a, b}$, V. Gori ${ }^{a, b}$, P. Lenzi ${ }^{a, b}$, M. Meschini ${ }^{a}$, S. Paoletti ${ }^{a}$, G. Sguazzoni ${ }^{a}$, A. Tropiano ${ }^{a, b}$

\section{INFN Laboratori Nazionali di Frascati, Frascati, Italy}

L. Benussi, S. Bianco, F. Fabbri, D. Piccolo

INFN Sezione di Genova ${ }^{a}$, Università di Genova ${ }^{b}$, Genova, Italy

P. Fabbricatore ${ }^{a}$, R. Ferretti ${ }^{a, b}$, F. Ferro ${ }^{a}$, M. Lo Vetere ${ }^{a, b}$, R. Musenich ${ }^{a}$, E. Robutti ${ }^{a}$, S. Tosi ${ }^{a, b}$

INFN Sezione di Milano-Bicocca ${ }^{a}$, Università di Milano-Bicocca ${ }^{b}$, Milano, Italy

A. Benaglia ${ }^{a}$, M.E. Dinardo ${ }^{a, b}$, S. Fiorendi ${ }^{a, b, 2}$, S. Gennai $^{a}$, A. Ghezzi $^{a, b}$, P. Govoni $^{a, b}$, M.T. Lucchini ${ }^{a, b, 2}$, S. Malvezzi ${ }^{a}$, R.A. Manzoni ${ }^{a, b, 2}$, A. Martelli ${ }^{a, b, 2}$, D. Menasce $^{a}$, L. Moroni ${ }^{a}$, M. Paganoni ${ }^{a, b}$, D. Pedrini ${ }^{a}$, S. Ragazzi ${ }^{a}, b$, N. Redaelli ${ }^{a}$, T. Tabarelli de Fatis $^{a, b}$ 
INFN Sezione di Napoli ${ }^{a}$, Università di Napoli 'Federico II' ${ }^{b}$, Università della Basilicata (Potenza) ${ }^{c}$, Università G. Marconi (Roma) ${ }^{d}$, Napoli, Italy

S. Buontempo ${ }^{a}$, N. Cavallo ${ }^{a, c}$, F. Fabozzi ${ }^{a, c}$, A.O.M. Iorio ${ }^{a, b}$, L. Lista $^{a}$, S. Meola ${ }^{a, d, 2}$, M. Merola ${ }^{a}$, P. Paolucci ${ }^{a}, 2$

INFN Sezione di Padova ${ }^{a}$, Università di Padova ${ }^{b}$, Università di Trento (Trento) ${ }^{c}$, Padova, Italy

P. Azzi $^{a}$, N. Bacchetta ${ }^{a}$, D. Bisello ${ }^{a, b}$, A. Branca ${ }^{a, b}$, R. Carlin ${ }^{a, b}$, P. Checchia ${ }^{a}$, T. Dorigo ${ }^{a}$, U. Dosselli ${ }^{a}$, M. Galanti ${ }^{a, b}{ }^{2}$, F. Gasparini ${ }^{a, b}$, U. Gasparini ${ }^{a, b}$, P. Giubilato $^{a, b}$,

A. Gozzelino ${ }^{a}$, K. Kanishchev ${ }^{a, c}$, S. Lacaprara ${ }^{a}$, I. Lazzizzera ${ }^{a, c}$, M. Margoni ${ }^{a, b}$, A.T. Meneguzzo ${ }^{a, b}$, M. Passaseo ${ }^{a}$, J. Pazzini ${ }^{a, b}$, M. Pegoraro ${ }^{a}$, N. Pozzobon ${ }^{a, b}$, P. Ronchese ${ }^{a, b}$, F. Simonetto ${ }^{a, b}$, E. Torassa ${ }^{a}$, M. Tosi ${ }^{a, b}$, S. Vanini ${ }^{a, b}$, P. Zotto $^{a, b}$, A. Zucchetta ${ }^{a, b}$, G. Zumerle ${ }^{a, b}$

INFN Sezione di Pavia ${ }^{a}$, Università di Pavia ${ }^{b}$, Pavia, Italy

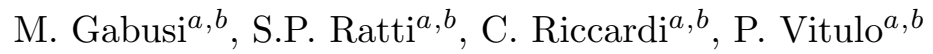

INFN Sezione di Perugia ${ }^{a}$, Università di Perugia ${ }^{b}$, Perugia, Italy

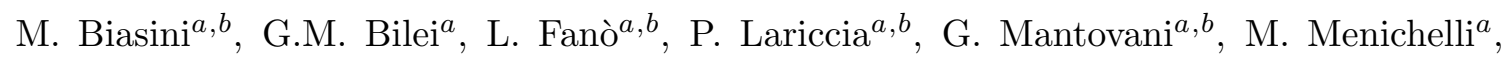
A. Nappi ${ }^{a, b \dagger}$, F. Romeo ${ }^{a, b}$, A. Saha ${ }^{a}$, A. Santocchia ${ }^{a, b}$, A. Spiezia ${ }^{a, b}$

INFN Sezione di Pisa ${ }^{a}$, Università di Pisa ${ }^{b}$, Scuola Normale Superiore di Pisa ${ }^{c}$, Pisa, Italy

K. Androsov ${ }^{a}, 29$, P. Azzurri ${ }^{a}$, G. Bagliesi ${ }^{a}$, J. Bernardini ${ }^{a}$, T. Boccali ${ }^{a}$, G. Broccolo $^{a, c}$, R. Castaldi ${ }^{a}$, M.A. Ciocci ${ }^{a, 29}$, R. Dell'Orso ${ }^{a}$, F. Fiori ${ }^{a, c}$, L. Foà ${ }^{a, c}$, A. Giassi ${ }^{a}$,

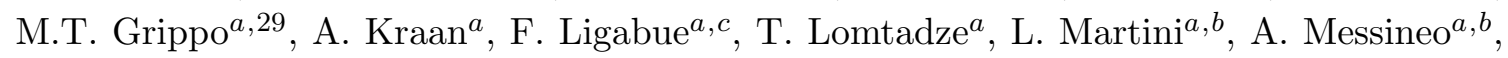
C.S. Moon ${ }^{a, 30}$, F. Palla ${ }^{a}$, A. Rizzi ${ }^{a, b}$, A. Savoy-Navarro ${ }^{a, 31}$, A.T. Serban ${ }^{a}$, P. Spagnolo ${ }^{a}$,

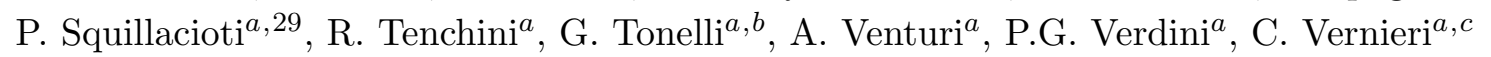

INFN Sezione di Roma ${ }^{a}$, Università di Roma ${ }^{b}$, Roma, Italy

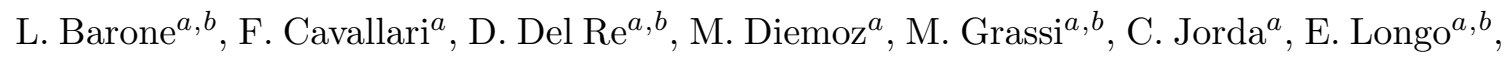
F. Margaroli ${ }^{a, b}$, P. Meridiani ${ }^{a}$, F. Micheli ${ }^{a, b}$, S. Nourbakhsh ${ }^{a, b}$, G. Organtini ${ }^{a, b}$, R. Paramatti ${ }^{a}$, S. Rahatlou ${ }^{a, b}$, C. Rovelli $^{a}$, L. Soffi $^{a, b}$, P. Traczyk $^{a, b}$

INFN Sezione di Torino ${ }^{a}$, Università di Torino ${ }^{b}$, Università del Piemonte Orientale (Novara) ${ }^{c}$, Torino, Italy

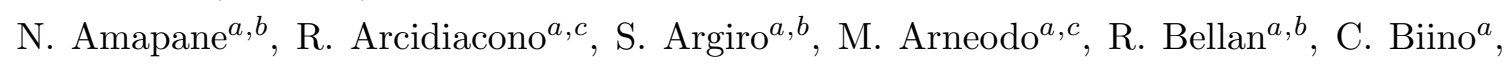
N. Cartiglia ${ }^{a}$, S. Casasso ${ }^{a, b}$, M. Costa ${ }^{a, b}$, A. Degano ${ }^{a, b}$, N. Demaria ${ }^{a}$, C. Mariotti ${ }^{a}$, S. Maselli ${ }^{a}$, E. Migliore ${ }^{a, b}$, V. Monaco ${ }^{a, b}$, M. Musich ${ }^{a}$, M.M. Obertino $^{a, c}$, G. Ortona ${ }^{a, b}$, L. Pacher ${ }^{a, b}$, N. Pastrone ${ }^{a}$, M. Pelliccioni ${ }^{a, 2}$, A. Potenza ${ }^{a, b}$, A. Romero $^{a, b}$, M. Ruspa $^{a, c}$, R. Sacchi ${ }^{a, b}$, A. Solano ${ }^{a, b}$, A. Staiano ${ }^{a}$, U. Tamponi $^{a}$

INFN Sezione di Trieste ${ }^{a}$, Università di Trieste ${ }^{b}$, Trieste, Italy

S. Belforte ${ }^{a}$, V. Candelise ${ }^{a, b}$, M. Casarsa ${ }^{a}$, F. Cossutti ${ }^{a, 2}$, G. Della Ricca ${ }^{a, b}$, B. Gobbo ${ }^{a}$,

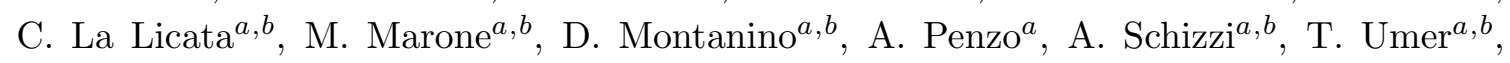
A. Zanetti ${ }^{a}$ 
Kangwon National University, Chunchon, Korea

S. Chang, T.Y. Kim, S.K. Nam

Kyungpook National University, Daegu, Korea

D.H. Kim, G.N. Kim, J.E. Kim, D.J. Kong, S. Lee, Y.D. Oh, H. Park, D.C. Son

Chonnam National University, Institute for Universe and Elementary Particles, Kwangju, Korea

J.Y. Kim, Zero J. Kim, S. Song

Korea University, Seoul, Korea

S. Choi, D. Gyun, B. Hong, M. Jo, H. Kim, Y. Kim, K.S. Lee, S.K. Park, Y. Roh

University of Seoul, Seoul, Korea

M. Choi, J.H. Kim, C. Park, I.C. Park, S. Park, G. Ryu

Sungkyunkwan University, Suwon, Korea

Y. Choi, Y.K. Choi, J. Goh, M.S. Kim, E. Kwon, B. Lee, J. Lee, S. Lee, H. Seo, I. Yu

Vilnius University, Vilnius, Lithuania

I. Grigelionis, A. Juodagalvis

Centro de Investigacion y de Estudios Avanzados del IPN, Mexico City, Mexico

H. Castilla-Valdez, E. De La Cruz-Burelo, I. Heredia-de La Cruz ${ }^{32}$, R. Lopez-Fernandez, J. Martínez-Ortega, A. Sanchez-Hernandez, L.M. Villasenor-Cendejas

Universidad Iberoamericana, Mexico City, Mexico

S. Carrillo Moreno, F. Vazquez Valencia

Benemerita Universidad Autonoma de Puebla, Puebla, Mexico

H.A. Salazar Ibarguen

Universidad Autónoma de San Luis Potosí, San Luis Potosí, Mexico

E. Casimiro Linares, A. Morelos Pineda

University of Auckland, Auckland, New Zealand

D. Krofcheck

University of Canterbury, Christchurch, New Zealand

P.H. Butler, R. Doesburg, S. Reucroft, H. Silverwood

National Centre for Physics, Quaid-I-Azam University, Islamabad, Pakistan

M. Ahmad, M.I. Asghar, J. Butt, H.R. Hoorani, S. Khalid, W.A. Khan, T. Khurshid, S. Qazi, M.A. Shah, M. Shoaib

National Centre for Nuclear Research, Swierk, Poland

H. Bialkowska, M. Bluj ${ }^{33}$, B. Boimska, T. Frueboes, M. Górski, M. Kazana, K. Nawrocki, K. Romanowska-Rybinska, M. Szleper, G. Wrochna, P. Zalewski 
Institute of Experimental Physics, Faculty of Physics, University of Warsaw, Warsaw, Poland

G. Brona, K. Bunkowski, M. Cwiok, W. Dominik, K. Doroba, A. Kalinowski, M. Konecki, J. Krolikowski, M. Misiura, W. Wolszczak

Laboratório de Instrumentação e Física Experimental de Partículas, Lisboa, Portugal

P. Bargassa, C. Beirão Da Cruz E Silva, P. Faccioli, P.G. Ferreira Parracho, M. Gallinaro, F. Nguyen, J. Rodrigues Antunes, J. Seixas ${ }^{2}$, J. Varela, P. Vischia

Joint Institute for Nuclear Research, Dubna, Russia

S. Afanasiev, I. Golutvin, I. Gorbunov, A. Kamenev, V. Karjavin, V. Konoplyanikov,

G. Kozlov, A. Lanev, A. Malakhov, V. Matveev, P. Moisenz, V. Palichik, V. Perelygin,

M. Savina, S. Shmatov, N. Skatchkov, V. Smirnov, A. Zarubin

Petersburg Nuclear Physics Institute, Gatchina (St. Petersburg), Russia

V. Golovtsov, Y. Ivanov, V. Kim, P. Levchenko, V. Murzin, V. Oreshkin, I. Smirnov, V. Sulimov, L. Uvarov, S. Vavilov, A. Vorobyev, An. Vorobyev

Institute for Nuclear Research, Moscow, Russia

Yu. Andreev, A. Dermenev, S. Gninenko, N. Golubev, M. Kirsanov, N. Krasnikov, A. Pashenkov, D. Tlisov, A. Toropin

Institute for Theoretical and Experimental Physics, Moscow, Russia

V. Epshteyn, V. Gavrilov, N. Lychkovskaya, V. Popov, G. Safronov, S. Semenov, A. Spiridonov, V. Stolin, E. Vlasov, A. Zhokin

P.N. Lebedev Physical Institute, Moscow, Russia

V. Andreev, M. Azarkin, I. Dremin, M. Kirakosyan, A. Leonidov, G. Mesyats, S.V. Rusakov, A. Vinogradov

Skobeltsyn Institute of Nuclear Physics, Lomonosov Moscow State University, Moscow, Russia

A. Belyaev, E. Boos, V. Bunichev, M. Dubinin ${ }^{7}$, L. Dudko, A. Ershov, A. Gribushin, V. Klyukhin, O. Kodolova, I. Lokhtin, A. Markina, S. Obraztsov, S. Petrushanko, V. Savrin

State Research Center of Russian Federation, Institute for High Energy Physics, Protvino, Russia

I. Azhgirey, I. Bayshev, S. Bitioukov, V. Kachanov, A. Kalinin, D. Konstantinov,

V. Krychkine, V. Petrov, R. Ryutin, A. Sobol, L. Tourtchanovitch, S. Troshin, N. Tyurin,

A. Uzunian, A. Volkov

University of Belgrade, Faculty of Physics and Vinca Institute of Nuclear Sciences, Belgrade, Serbia

P. Adzic ${ }^{34}$, M. Djordjevic, M. Ekmedzic, J. Milosevic 
Centro de Investigaciones Energéticas Medioambientales y Tecnológicas (CIEMAT), Madrid, Spain

M. Aguilar-Benitez, J. Alcaraz Maestre, C. Battilana, E. Calvo, M. Cerrada, M. Chamizo Llatas $^{2}$, N. Colino, B. De La Cruz, A. Delgado Peris, D. Domínguez Vázquez, C. Fernandez Bedoya, J.P. Fernández Ramos, A. Ferrando, J. Flix, M.C. Fouz, P. Garcia-Abia, O. Gonzalez Lopez, S. Goy Lopez, J.M. Hernandez, M.I. Josa, G. Merino, E. Navarro De Martino, J. Puerta Pelayo, A. Quintario Olmeda, I. Redondo, L. Romero, M.S. Soares, C. Willmott

Universidad Autónoma de Madrid, Madrid, Spain

C. Albajar, J.F. de Trocóniz

\section{Universidad de Oviedo, Oviedo, Spain}

H. Brun, J. Cuevas, J. Fernandez Menendez, S. Folgueras, I. Gonzalez Caballero, L. Lloret Iglesias

Instituto de Física de Cantabria (IFCA), CSIC-Universidad de Cantabria, Santander, Spain

J.A. Brochero Cifuentes, I.J. Cabrillo, A. Calderon, S.H. Chuang, J. Duarte Campderros, M. Fernandez, G. Gomez, J. Gonzalez Sanchez, A. Graziano, A. Lopez Virto, J. Marco, R. Marco, C. Martinez Rivero, F. Matorras, F.J. Munoz Sanchez, J. Piedra Gomez, T. Rodrigo, A.Y. Rodríguez-Marrero, A. Ruiz-Jimeno, L. Scodellaro, I. Vila, R. Vilar Cortabitarte

\section{CERN, European Organization for Nuclear Research, Geneva, Switzerland}

D. Abbaneo, E. Auffray, G. Auzinger, M. Bachtis, P. Baillon, A.H. Ball, D. Barney, J. Bendavid, L. Benhabib, J.F. Benitez, C. Bernet ${ }^{8}$, G. Bianchi, P. Bloch, A. Bocci, A. Bonato, O. Bondu, C. Botta, H. Breuker, T. Camporesi, G. Cerminara, T. Christiansen, J.A. Coarasa Perez, S. Colafranceschi ${ }^{35}$, M. D'Alfonso, D. d'Enterria, A. Dabrowski, A. David, F. De Guio, A. De Roeck, S. De Visscher, S. Di Guida, M. Dobson, N. DupontSagorin, A. Elliott-Peisert, J. Eugster, G. Franzoni, W. Funk, M. Giffels, D. Gigi, K. Gill, M. Girone, M. Giunta, F. Glege, R. Gomez-Reino Garrido, S. Gowdy, R. Guida, J. Hammer, M. Hansen, P. Harris, A. Hinzmann, V. Innocente, P. Janot, E. Karavakis, K. Kousouris, K. Krajczar, P. Lecoq, Y.-J. Lee, C. Lourenço, N. Magini, L. Malgeri, M. Mannelli, L. Masetti, F. Meijers, S. Mersi, E. Meschi, F. Moortgat, M. Mulders, P. Musella, L. Orsini, E. Palencia Cortezon, E. Perez, L. Perrozzi, A. Petrilli, G. Petrucciani, A. Pfeiffer, M. Pierini, M. Pimiä, D. Piparo, M. Plagge, A. Racz, W. Reece, G. Rolandi ${ }^{36}$, M. Rovere, H. Sakulin, F. Santanastasio, C. Schäfer, C. Schwick, S. Sekmen, A. Sharma, P. Siegrist, P. Silva, M. Simon, P. Sphicas ${ }^{37}$, J. Steggemann, B. Stieger, M. Stoye, A. Tsirou, G.I. Veres ${ }^{20}$, J.R. Vlimant, H.K. Wöhri, W.D. Zeuner

\section{Paul Scherrer Institut, Villigen, Switzerland}

W. Bertl, K. Deiters, W. Erdmann, K. Gabathuler, R. Horisberger, Q. Ingram, H.C. Kaestli, S. König, D. Kotlinski, U. Langenegger, D. Renker, T. Rohe 
Institute for Particle Physics, ETH Zurich, Zurich, Switzerland

F. Bachmair, L. Bäni, L. Bianchini, P. Bortignon, M.A. Buchmann, B. Casal, N. Chanon, A. Deisher, G. Dissertori, M. Dittmar, M. Donegà, M. Dünser, P. Eller, C. Grab, D. Hits, W. Lustermann, B. Mangano, A.C. Marini, P. Martinez Ruiz del Arbol, D. Meister, N. Mohr, C. Nägeli ${ }^{38}$, P. Nef, F. Nessi-Tedaldi, F. Pandolfi, L. Pape, F. Pauss, M. Peruzzi, M. Quittnat, F.J. Ronga, M. Rossini, L. Sala, A. Starodumov ${ }^{39}$, M. Takahashi, L. Tauscher ${ }^{\dagger}$, K. Theofilatos, D. Treille, R. Wallny, H.A. Weber

Universität Zürich, Zurich, Switzerland

C. Amsler ${ }^{40}$, V. Chiochia, A. De Cosa, C. Favaro, M. Ivova Rikova, B. Kilminster, B. Millan Mejias, J. Ngadiuba, P. Robmann, H. Snoek, S. Taroni, M. Verzetti, Y. Yang

National Central University, Chung-Li, Taiwan

M. Cardaci, K.H. Chen, C. Ferro, C.M. Kuo, S.W. Li, W. Lin, Y.J. Lu, R. Volpe, S.S. Yu National Taiwan University (NTU), Taipei, Taiwan

P. Bartalini, P. Chang, Y.H. Chang, Y.W. Chang, Y. Chao, K.F. Chen, C. Dietz, U. Grundler, W.-S. Hou, Y. Hsiung, K.Y. Kao, Y.J. Lei, Y.F. Liu, R.-S. Lu, D. Majumder, E. Petrakou, X. Shi, J.G. Shiu, Y.M. Tzeng, M. Wang, R. Wilken

Chulalongkorn University, Bangkok, Thailand

B. Asavapibhop, N. Suwonjandee

Cukurova University, Adana, Turkey

A. Adiguzel, M.N. Bakirci ${ }^{41}$, S. Cerci ${ }^{42}$, C. Dozen, I. Dumanoglu, E. Eskut, S. Girgis, G. Gokbulut, E. Gurpinar, I. Hos, E.E. Kangal, A. Kayis Topaksu, G. Onengut ${ }^{43}$, K. Ozdemir, S. Ozturk ${ }^{41}$, A. Polatoz, K. Sogut ${ }^{44}$, D. Sunar Cerci ${ }^{42}$, B. Tali ${ }^{42}$, H. Topakli ${ }^{41}$, M. Vergili

Middle East Technical University, Physics Department, Ankara, Turkey

I.V. Akin, T. Aliev, B. Bilin, S. Bilmis, M. Deniz, H. Gamsizkan, A.M. Guler, G. Karapinar ${ }^{45}$, K. Ocalan, A. Ozpineci, M. Serin, R. Sever, U.E. Surat, M. Yalvac, M. Zeyrek

Bogazici University, Istanbul, Turkey

E. Gülmez, B. Isildak ${ }^{46}$, M. Kaya ${ }^{47}$, O. Kaya ${ }^{47}$, S. Ozkorucuklu ${ }^{48}$, N. Sonmez ${ }^{49}$

Istanbul Technical University, Istanbul, Turkey

H. Bahtiyar ${ }^{50}$, E. Barlas, K. Cankocak, Y.O. Günaydin ${ }^{51}$, F.I. Vardarl, M. Yücel

National Scientific Center, Kharkov Institute of Physics and Technology, Kharkov, Ukraine

L. Levchuk, P. Sorokin

University of Bristol, Bristol, United Kingdom

J.J. Brooke, E. Clement, D. Cussans, H. Flacher, R. Frazier, J. Goldstein, M. Grimes, G.P. Heath, H.F. Heath, J. Jacob, L. Kreczko, C. Lucas, Z. Meng, S. Metson, D.M. Newbold ${ }^{52}$, K. Nirunpong, S. Paramesvaran, A. Poll, S. Senkin, V.J. Smith, T. Williams 


\section{Rutherford Appleton Laboratory, Didcot, United Kingdom}

K.W. Bell, A. Belyaev ${ }^{53}$, C. Brew, R.M. Brown, D.J.A. Cockerill, J.A. Coughlan, K. Harder, S. Harper, J. Ilic, E. Olaiya, D. Petyt, C.H. Shepherd-Themistocleous, A. Thea, I.R. Tomalin, W.J. Womersley, S.D. Worm

\section{Imperial College, London, United Kingdom}

M. Baber, R. Bainbridge, O. Buchmuller, D. Burton, D. Colling, N. Cripps, M. Cutajar, P. Dauncey, G. Davies, M. Della Negra, W. Ferguson, J. Fulcher, D. Futyan, A. Gilbert, A. Guneratne Bryer, G. Hall, Z. Hatherell, J. Hays, G. Iles, M. Jarvis, G. Karapostoli, M. Kenzie, R. Lane, R. Lucas ${ }^{52}$, L. Lyons, A.-M. Magnan, J. Marrouche, B. Mathias, R. Nandi, J. Nash, A. Nikitenko ${ }^{39}$, J. Pela, M. Pesaresi, K. Petridis, M. Pioppi ${ }^{54}$, D.M. Raymond, S. Rogerson, A. Rose, C. Seez, P. Sharp ${ }^{\dagger}$, A. Sparrow, A. Tapper, M. Vazquez Acosta, T. Virdee, S. Wakefield, N. Wardle

\section{Brunel University, Uxbridge, United Kingdom}

J.E. Cole, P.R. Hobson, A. Khan, P. Kyberd, D. Leggat, D. Leslie, W. Martin, I.D. Reid, P. Symonds, L. Teodorescu, M. Turner

\section{Baylor University, Waco, USA}

J. Dittmann, K. Hatakeyama, A. Kasmi, H. Liu, T. Scarborough

The University of Alabama, Tuscaloosa, USA

O. Charaf, S.I. Cooper, C. Henderson, P. Rumerio

\section{Boston University, Boston, USA}

A. Avetisyan, T. Bose, C. Fantasia, A. Heister, P. Lawson, D. Lazic, J. Rohlf, D. Sperka, J. St. John, L. Sulak

\section{Brown University, Providence, USA}

J. Alimena, S. Bhattacharya, G. Christopher, D. Cutts, Z. Demiragli, A. Ferapontov, A. Garabedian, U. Heintz, S. Jabeen, G. Kukartsev, E. Laird, G. Landsberg, M. Luk, M. Narain, M. Segala, T. Sinthuprasith, T. Speer

\section{University of California, Davis, Davis, USA}

R. Breedon, G. Breto, M. Calderon De La Barca Sanchez, S. Chauhan, M. Chertok, J. Conway, R. Conway, P.T. Cox, R. Erbacher, M. Gardner, W. Ko, A. Kopecky, R. Lander, T. Miceli, D. Pellett, J. Pilot, F. Ricci-Tam, B. Rutherford, M. Searle, S. Shalhout, J. Smith, M. Squires, M. Tripathi, S. Wilbur, R. Yohay

\section{University of California, Los Angeles, USA}

V. Andreev, D. Cline, R. Cousins, S. Erhan, P. Everaerts, C. Farrell, M. Felcini, J. Hauser, M. Ignatenko, C. Jarvis, G. Rakness, P. Schlein ${ }^{\dagger}$, E. Takasugi, V. Valuev, M. Weber

\section{University of California, Riverside, Riverside, USA}

J. Babb, R. Clare, J. Ellison, J.W. Gary, G. Hanson, J. Heilman, P. Jandir, F. Lacroix, H. Liu, O.R. Long, A. Luthra, M. Malberti, H. Nguyen, A. Shrinivas, J. Sturdy, S. Sumowidagdo, S. Wimpenny 


\section{University of California, San Diego, La Jolla, USA}

W. Andrews, J.G. Branson, G.B. Cerati, S. Cittolin, R.T. D’Agnolo, D. Evans, A. Holzner, R. Kelley, D. Kovalskyi, M. Lebourgeois, J. Letts, I. Macneill, S. Padhi, C. Palmer, M. Pieri, M. Sani, V. Sharma, S. Simon, E. Sudano, M. Tadel, Y. Tu, A. Vartak, S. Wasserbaech ${ }^{55}$, F. Würthwein, A. Yagil, J. Yoo

\section{University of California, Santa Barbara, Santa Barbara, USA}

D. Barge, C. Campagnari, T. Danielson, K. Flowers, P. Geffert, C. George, F. Golf, J. Incandela, C. Justus, R. Magaña Villalba, N. Mccoll, V. Pavlunin, J. Richman, R. Rossin, D. Stuart, W. To, C. West

\section{California Institute of Technology, Pasadena, USA}

A. Apresyan, A. Bornheim, J. Bunn, Y. Chen, E. Di Marco, J. Duarte, D. Kcira, Y. Ma, A. Mott, H.B. Newman, C. Pena, C. Rogan, M. Spiropulu, V. Timciuc, R. Wilkinson, S. Xie, R.Y. Zhu

\section{Carnegie Mellon University, Pittsburgh, USA}

V. Azzolini, A. Calamba, R. Carroll, T. Ferguson, Y. Iiyama, D.W. Jang, M. Paulini, J. Russ, H. Vogel, I. Vorobiev

\section{University of Colorado at Boulder, Boulder, USA}

J.P. Cumalat, B.R. Drell, W.T. Ford, A. Gaz, E. Luiggi Lopez, U. Nauenberg, J.G. Smith, K. Stenson, K.A. Ulmer, S.R. Wagner

\section{Cornell University, Ithaca, USA}

J. Alexander, A. Chatterjee, N. Eggert, L.K. Gibbons, W. Hopkins, A. Khukhunaishvili, B. Kreis, N. Mirman, G. Nicolas Kaufman, J.R. Patterson, A. Ryd, E. Salvati, W. Sun, W.D. Teo, J. Thom, J. Thompson, J. Tucker, Y. Weng, L. Winstrom, P. Wittich

\section{Fairfield University, Fairfield, USA}

D. Winn

\section{Fermi National Accelerator Laboratory, Batavia, USA}

S. Abdullin, M. Albrow, J. Anderson, G. Apollinari, L.A.T. Bauerdick, A. Beretvas, J. Berryhill, P.C. Bhat, K. Burkett, J.N. Butler, V. Chetluru, H.W.K. Cheung, F. Chlebana, S. Cihangir, V.D. Elvira, I. Fisk, J. Freeman, Y. Gao, E. Gottschalk, L. Gray, D. Green, O. Gutsche, D. Hare, R.M. Harris, J. Hirschauer, B. Hooberman, S. Jindariani, M. Johnson, U. Joshi, K. Kaadze, B. Klima, S. Kwan, J. Linacre, D. Lincoln, R. Lipton, J. Lykken, K. Maeshima, J.M. Marraffino, V.I. Martinez Outschoorn, S. Maruyama, D. Mason, P. McBride, K. Mishra, S. Mrenna, Y. Musienko ${ }^{56}$, S. Nahn, C. Newman-Holmes, V. O’Dell, O. Prokofyev, N. Ratnikova, E. Sexton-Kennedy, S. Sharma, W.J. Spalding, L. Spiegel, L. Taylor, S. Tkaczyk, N.V. Tran, L. Uplegger, E.W. Vaandering, R. Vidal, J. Whitmore, W. Wu, F. Yang, J.C. Yun

\section{University of Florida, Gainesville, USA}

D. Acosta, P. Avery, D. Bourilkov, T. Cheng, S. Das, M. De Gruttola, G.P. Di Giovanni, D. Dobur, R.D. Field, M. Fisher, Y. Fu, I.K. Furic, J. Hugon, B. Kim, J. Konigsberg, 
A. Korytov, A. Kropivnitskaya, T. Kypreos, J.F. Low, K. Matchev, P. Milenovic ${ }^{57}$, G. Mitselmakher, L. Muniz, A. Rinkevicius, L. Shchutska, N. Skhirtladze, M. Snowball, J. Yelton, M. Zakaria

Florida International University, Miami, USA

V. Gaultney, S. Hewamanage, S. Linn, P. Markowitz, G. Martinez, J.L. Rodriguez

Florida State University, Tallahassee, USA

T. Adams, A. Askew, J. Bochenek, J. Chen, B. Diamond, J. Haas, S. Hagopian, V. Hagopian, K.F. Johnson, H. Prosper, V. Veeraraghavan, M. Weinberg

Florida Institute of Technology, Melbourne, USA

M.M. Baarmand, B. Dorney, M. Hohlmann, H. Kalakhety, F. Yumiceva

University of Illinois at Chicago (UIC), Chicago, USA

M.R. Adams, L. Apanasevich, V.E. Bazterra, R.R. Betts, I. Bucinskaite, R. Cavanaugh, O. Evdokimov, L. Gauthier, C.E. Gerber, D.J. Hofman, S. Khalatyan, P. Kurt, D.H. Moon,

C. O'Brien, C. Silkworth, P. Turner, N. Varelas

The University of Iowa, Iowa City, USA

U. Akgun, E.A. Albayrak ${ }^{50}$, B. Bilki ${ }^{58}$, W. Clarida, K. Dilsiz, F. Duru, J.-P. Merlo, H. Mermerkaya ${ }^{59}$, A. Mestvirishvili, A. Moeller, J. Nachtman, H. Ogul, Y. Onel, F. Ozok ${ }^{50}$, S. Sen, P. Tan, E. Tiras, J. Wetzel, T. Yetkin ${ }^{60}$, K. Yi

Johns Hopkins University, Baltimore, USA

B.A. Barnett, B. Blumenfeld, S. Bolognesi, D. Fehling, A.V. Gritsan, P. Maksimovic, C. Martin, M. Swartz, A. Whitbeck

The University of Kansas, Lawrence, USA

P. Baringer, A. Bean, G. Benelli, R.P. Kenny III, M. Murray, D. Noonan, S. Sanders, J. Sekaric, R. Stringer, Q. Wang, J.S. Wood

Kansas State University, Manhattan, USA

A.F. Barfuss, I. Chakaberia, A. Ivanov, S. Khalil, M. Makouski, Y. Maravin, L.K. Saini, S. Shrestha, I. Svintradze

Lawrence Livermore National Laboratory, Livermore, USA

J. Gronberg, D. Lange, F. Rebassoo, D. Wright

University of Maryland, College Park, USA

A. Baden, B. Calvert, S.C. Eno, J.A. Gomez, N.J. Hadley, R.G. Kellogg, T. Kolberg, Y. Lu, M. Marionneau, A.C. Mignerey, K. Pedro, A. Skuja, J. Temple, M.B. Tonjes, S.C. Tonwar

Massachusetts Institute of Technology, Cambridge, USA

A. Apyan, G. Bauer, W. Busza, I.A. Cali, M. Chan, L. Di Matteo, V. Dutta, G. Gomez Ceballos, M. Goncharov, D. Gulhan, M. Klute, Y.S. Lai, A. Levin, P.D. Luckey, T. Ma, C. Paus, D. Ralph, C. Roland, G. Roland, G.S.F. Stephans, F. Stöckli, K. Sumorok, D. Velicanu, J. Veverka, B. Wyslouch, M. Yang, A.S. Yoon, M. Zanetti, V. Zhukova 


\section{University of Minnesota, Minneapolis, USA}

B. Dahmes, A. De Benedetti, A. Gude, S.C. Kao, K. Klapoetke, Y. Kubota, J. Mans,

N. Pastika, R. Rusack, A. Singovsky, N. Tambe, J. Turkewitz

\section{University of Mississippi, Oxford, USA}

J.G. Acosta, L.M. Cremaldi, R. Kroeger, S. Oliveros, L. Perera, R. Rahmat, D.A. Sanders, D. Summers

\section{University of Nebraska-Lincoln, Lincoln, USA}

E. Avdeeva, K. Bloom, S. Bose, D.R. Claes, A. Dominguez, R. Gonzalez Suarez, J. Keller, I. Kravchenko, J. Lazo-Flores, S. Malik, F. Meier, G.R. Snow

State University of New York at Buffalo, Buffalo, USA

J. Dolen, A. Godshalk, I. Iashvili, S. Jain, A. Kharchilava, A. Kumar, S. Rappoccio, Z. Wan

\section{Northeastern University, Boston, USA}

G. Alverson, E. Barberis, D. Baumgartel, M. Chasco, J. Haley, A. Massironi, D. Nash,

T. Orimoto, D. Trocino, D. Wood, J. Zhang

Northwestern University, Evanston, USA

A. Anastassov, K.A. Hahn, A. Kubik, L. Lusito, N. Mucia, N. Odell, B. Pollack, A. Pozdnyakov, M. Schmitt, S. Stoynev, K. Sung, M. Velasco, S. Won

University of Notre Dame, Notre Dame, USA

D. Berry, A. Brinkerhoff, K.M. Chan, A. Drozdetskiy, M. Hildreth, C. Jessop, D.J. Karmgard, J. Kolb, K. Lannon, W. Luo, S. Lynch, N. Marinelli, D.M. Morse, T. Pearson, M. Planer, R. Ruchti, J. Slaunwhite, N. Valls, M. Wayne, M. Wolf

\section{The Ohio State University, Columbus, USA}

L. Antonelli, B. Bylsma, L.S. Durkin, S. Flowers, C. Hill, R. Hughes, K. Kotov, T.Y. Ling,

D. Puigh, M. Rodenburg, G. Smith, C. Vuosalo, B.L. Winer, H. Wolfe, H.W. Wulsin

\section{Princeton University, Princeton, USA}

E. Berry, P. Elmer, V. Halyo, P. Hebda, J. Hegeman, A. Hunt, P. Jindal, S.A. Koay, P. Lujan, D. Marlow, T. Medvedeva, M. Mooney, J. Olsen, P. Piroué, X. Quan, A. Raval, H. Saka, D. Stickland, C. Tully, J.S. Werner, S.C. Zenz, A. Zuranski

\section{University of Puerto Rico, Mayaguez, USA}

E. Brownson, A. Lopez, H. Mendez, J.E. Ramirez Vargas

\section{Purdue University, West Lafayette, USA}

E. Alagoz, D. Benedetti, G. Bolla, D. Bortoletto, M. De Mattia, A. Everett, Z. Hu, M. Jones, K. Jung, M. Kress, N. Leonardo, D. Lopes Pegna, V. Maroussov, P. Merkel, D.H. Miller, N. Neumeister, B.C. Radburn-Smith, I. Shipsey, D. Silvers, A. Svyatkovskiy, F. Wang, W. Xie, L. Xu, H.D. Yoo, J. Zablocki, Y. Zheng

\section{Purdue University Calumet, Hammond, USA}

N. Parashar 


\section{Rice University, Houston, USA}

A. Adair, B. Akgun, K.M. Ecklund, F.J.M. Geurts, W. Li, B. Michlin, B.P. Padley, R. Redjimi, J. Roberts, J. Zabel

\section{University of Rochester, Rochester, USA}

B. Betchart, A. Bodek, R. Covarelli, P. de Barbaro, R. Demina, Y. Eshaq, T. Ferbel, A. Garcia-Bellido, P. Goldenzweig, J. Han, A. Harel, D.C. Miner, G. Petrillo, D. Vishnevskiy, M. Zielinski

The Rockefeller University, New York, USA

A. Bhatti, R. Ciesielski, L. Demortier, K. Goulianos, G. Lungu, S. Malik, C. Mesropian

Rutgers, The State University of New Jersey, Piscataway, USA

S. Arora, A. Barker, J.P. Chou, C. Contreras-Campana, E. Contreras-Campana, D. Duggan, D. Ferencek, Y. Gershtein, R. Gray, E. Halkiadakis, D. Hidas, A. Lath, S. Panwalkar, M. Park, R. Patel, V. Rekovic, J. Robles, S. Salur, S. Schnetzer, C. Seitz, S. Somalwar, R. Stone, S. Thomas, P. Thomassen, M. Walker

University of Tennessee, Knoxville, USA

K. Rose, S. Spanier, Z.C. Yang, A. York

Texas A\&M University, College Station, USA

O. Bouhali ${ }^{61}$, R. Eusebi, W. Flanagan, J. Gilmore, T. Kamon ${ }^{62}$, V. Khotilovich, V. Krutelyov, R. Montalvo, I. Osipenkov, Y. Pakhotin, A. Perloff, J. Roe, A. Safonov, T. Sakuma, I. Suarez, A. Tatarinov, D. Toback

\section{Texas Tech University, Lubbock, USA}

N. Akchurin, C. Cowden, J. Damgov, C. Dragoiu, P.R. Dudero, K. Kovitanggoon, S. Kunori, S.W. Lee, T. Libeiro, I. Volobouev

\section{Vanderbilt University, Nashville, USA}

E. Appelt, A.G. Delannoy, S. Greene, A. Gurrola, W. Johns, C. Maguire, Y. Mao, A. Melo, M. Sharma, P. Sheldon, B. Snook, S. Tuo, J. Velkovska

\section{University of Virginia, Charlottesville, USA}

M.W. Arenton, S. Boutle, B. Cox, B. Francis, J. Goodell, R. Hirosky, A. Ledovskoy, C. Lin, C. Neu, J. Wood

\section{Wayne State University, Detroit, USA}

S. Gollapinni, R. Harr, P.E. Karchin, C. Kottachchi Kankanamge Don, P. Lamichhane, A. Sakharov

\section{University of Wisconsin, Madison, USA}

D.A. Belknap, L. Borrello, D. Carlsmith, M. Cepeda, S. Dasu, S. Duric, E. Friis, M. Grothe, R. Hall-Wilton, M. Herndon, A. Hervé, P. Klabbers, J. Klukas, A. Lanaro, R. Loveless, A. Mohapatra, I. Ojalvo, T. Perry, G.A. Pierro, G. Polese, I. Ross, T. Sarangi, A. Savin, W.H. Smith, J. Swanson 
$\dagger$ : Deceased

1: Also at Vienna University of Technology, Vienna, Austria

2: Also at CERN, European Organization for Nuclear Research, Geneva, Switzerland

3: Also at Institut Pluridisciplinaire Hubert Curien, Université de Strasbourg, Université de Haute Alsace Mulhouse, CNRS/IN2P3, Strasbourg, France

4: Also at National Institute of Chemical Physics and Biophysics, Tallinn, Estonia

5: Also at Skobeltsyn Institute of Nuclear Physics, Lomonosov Moscow State University, Moscow, Russia

6: Also at Universidade Estadual de Campinas, Campinas, Brazil

7: Also at California Institute of Technology, Pasadena, USA

8: Also at Laboratoire Leprince-Ringuet, Ecole Polytechnique, IN2P3-CNRS, Palaiseau, France

9: Also at Zewail City of Science and Technology, Zewail, Egypt

10: Also at Suez Canal University, Suez, Egypt

11: Also at Cairo University, Cairo, Egypt

12: Also at Fayoum University, El-Fayoum, Egypt

13: Also at British University in Egypt, Cairo, Egypt

14: Now at Ain Shams University, Cairo, Egypt

15: Also at Université de Haute Alsace, Mulhouse, France

16: Also at Universidad de Antioquia, Medellin, Colombia

17: Also at Brandenburg University of Technology, Cottbus, Germany

18: Also at The University of Kansas, Lawrence, USA

19: Also at Institute of Nuclear Research ATOMKI, Debrecen, Hungary

20: Also at Eötvös Loránd University, Budapest, Hungary

21: Also at Tata Institute of Fundamental Research - EHEP, Mumbai, India

22: Also at Tata Institute of Fundamental Research - HECR, Mumbai, India

23: Now at King Abdulaziz University, Jeddah, Saudi Arabia

24: Also at University of Visva-Bharati, Santiniketan, India

25: Also at University of Ruhuna, Matara, Sri Lanka

26: Also at Isfahan University of Technology, Isfahan, Iran

27: Also at Sharif University of Technology, Tehran, Iran

28: Also at Plasma Physics Research Center, Science and Research Branch, Islamic Azad University, Tehran, Iran

29: Also at Università degli Studi di Siena, Siena, Italy

30: Also at Centre National de la Recherche Scientifique (CNRS) - IN2P3, Paris, France

31: Also at Purdue University, West Lafayette, USA

32: Also at Universidad Michoacana de San Nicolas de Hidalgo, Morelia, Mexico

33: Also at National Centre for Nuclear Research, Swierk, Poland

34: Also at Faculty of Physics, University of Belgrade, Belgrade, Serbia

35: Also at Facoltà Ingegneria, Università di Roma, Roma, Italy

36: Also at Scuola Normale e Sezione dell'INFN, Pisa, Italy

37: Also at University of Athens, Athens, Greece

38: Also at Paul Scherrer Institut, Villigen, Switzerland

39: Also at Institute for Theoretical and Experimental Physics, Moscow, Russia

40: Also at Albert Einstein Center for Fundamental Physics, Bern, Switzerland

41: Also at Gaziosmanpasa University, Tokat, Turkey

42: Also at Adiyaman University, Adiyaman, Turkey

43: Also at Cag University, Mersin, Turkey

44: Also at Mersin University, Mersin, Turkey 
45: Also at Izmir Institute of Technology, Izmir, Turkey

46: Also at Ozyegin University, Istanbul, Turkey

47: Also at Kafkas University, Kars, Turkey

48: Also at Suleyman Demirel University, Isparta, Turkey

49: Also at Ege University, Izmir, Turkey

50: Also at Mimar Sinan University, Istanbul, Istanbul, Turkey

51: Also at Kahramanmaras Sütcü Imam University, Kahramanmaras, Turkey

52: Also at Rutherford Appleton Laboratory, Didcot, United Kingdom

53: Also at School of Physics and Astronomy, University of Southampton, Southampton, United Kingdom

54: Also at INFN Sezione di Perugia; Università di Perugia, Perugia, Italy

55: Also at Utah Valley University, Orem, USA

56: Also at Institute for Nuclear Research, Moscow, Russia

57: Also at University of Belgrade, Faculty of Physics and Vinca Institute of Nuclear Sciences, Belgrade, Serbia

58: Also at Argonne National Laboratory, Argonne, USA

59: Also at Erzincan University, Erzincan, Turkey

60: Also at Yildiz Technical University, Istanbul, Turkey

61: Also at Texas A\&M University at Qatar, Doha, Qatar

62: Also at Kyungpook National University, Daegu, Korea 